\title{
Reproducibility of mild to moderate regurgitation of all heart valves by magnetic resonance imaging
}

\author{
Robert Gradinger ${ }^{*}$, Christina Ring, Thomas Walcher, Wolfgang Rottbauer, Peter Bernhardt \\ From 2011 SCMR/Euro CMR Joint Scientific Sessions \\ Nice, France. 3-6 February 2011
}

\section{Introduction}

Cardiac magnetic resonance imaging (CMR) is regarded as the gold standard for evaluation of left and right ventricular volumes and ejection fraction. Assessment of valve regurgitation can be performed by flow measurement in the great arteries for the aortic and pulmonary valve. Subtraction of ventricle stroke volume and flow measurement in the affiliated great artery results in regurgitation volume of the corresponding atrioventricular valve.

Aim of this study was to evaluate the reproducibility of valve regurgitations considering intra- and interobserver variability in patients with mild to moderate valve regurgitation.

\section{Methods}

For sample size estimation two blinded observers analyzed twice the CMR data of ten patients. To yield a confidence interval bandwidth of 0.15 twenty-six patients had to be evaluated by two blinded observers. All images were analyzed by two experienced readers blinded to patients' data in random order. All patients were analyzed twice by every reader.

\section{Results}

We found good correlations for aortic and pulmonary regurgitation fraction and left ventricular ejection fraction (ICC > 0.9). Correlations for mitral and tricuspid valve regurgitation and right ventricular ejection fraction were lower. Right ventricular ejection fraction: inter-rater reliability: ICC 0.843 ; intra-rater reliability: rater 1 ICC 0.884; rater 2 ICC 0.840. Mitral regurgitation: inter-rater reliability: ICC 0.875; intra-rater reliability: rater 1 ICC 0.824; rater 2 ICC 0.849. Tricuspid regurgitation: inter-rater reliability: ICC 0.616 ; intra-rater reliability: rater 1 ICC 0.585; rater 2 ICC 0.787.

Universitiy of Ulm, Ulm, Germany

(c) 2011 Gradinger et al; licensee BioMed Central Ltd. This is an open access article distributed under the terms of the Creative Commons Attribution License (http://creativecommons.org/licenses/by/2.0), which permits unrestricted use, distribution, and reproduction in any medium, provided the original work is properly cited.

\section{Concusion}

Cardiac magnetic resonance imaging offers good to very good inter- and intrastudy agreement for the analysis of mild heart valve regurgitation. However, the exact evaluation of atrioventricular valve regurgitation, especially of the tricuspid valve yield lower inter- and intraobserver agreement. The reason might be low the inter- and intraobserver variability in the calculation of the right ventricular stroke volume, caused by the more complex function and anatomy of the right heart chambers.

Published: 2 February 2011

doi:10.1186/1532-429X-13-S1-P340

Cite this article as: Gradinger et al:: Reproducibility of mild to moderate regurgitation of all heart valves by magnetic resonance imaging. Journal of Cardiovascular Magnetic Resonance 2011 13(Suppl 1):P340. and take full advantage of:

- Convenient online submission

- Thorough peer review

- No space constraints or color figure charges

- Immediate publication on acceptance

- Inclusion in PubMed, CAS, Scopus and Google Scholar

- Research which is freely available for redistribution 